\title{
Design Comparison of Strut Tie Model and ACI Traditional by Clear Span-to-Depth Ratio
}

\author{
Uy Lymei ${ }^{1}$ and Byung-Jik Son ${ }^{1 *}$ \\ ${ }^{1}$ Dept of Civil and Environmental Engineering, Konyang University \\ 지간-높이 비에 따른 스트럿-타이 모델과 ACI 고전적인 방법의 \\ 설계 비교 \\ 오이 리마이 ${ }^{1}$, 손병직 ${ }^{1 *}$ \\ ${ }^{1}$ 건양대학교 건설환경공학과
}

\begin{abstract}
Since clear span-to-depth ratio is used to define what is so called a deep beam, it is an important parameter ratio for study about deep beam. Deep beams can be designed by flexure design method, and shear provided by concrete $\left(v_{c}\right)$ and by steel $\left(v_{s}\right)$ for deep flexure members are provided in ACI 318-99 [1]. But in later version of ACI (from ACI 318-02) it is not provided and deep beams shall be designed either by taking into account nonlinear distribution of strain or by Appendix A of Strut-and-Tie Models (STM). The trend of deep beam design seems to be familiar with strut-and-tie model, but ACI traditional design is not forgotten. By comparing these two method, there should a point which definitely explain the different between the two methods. In this study, 68 samples result of steel, after reinforcement arrangement, are taken to be analyzed.

요 약 깊은 보는 지간-높이 비가 중요한 파라미터이다. 깊은 보를 설계할 때, ACI 318-02 이후 버전에서는 응력의 비선형 분포로 인해서 고전적인 설계 방법이 아니라 스트럿-타이 모델을 부록에 수록하여, 이 방법으로 설계하도록 하고 있다. 본 연구에서는 $\mathrm{ACI}$ 고전적인 설계방법과 스트럿-타이 모델을 비교, 분석하였다. 파라미터 연구로 지간-높이 비를 변화시켰으 며, 총 68개의 샘플을 분석하였다. 결과로 고전적인 설계 방법과 스트럿-타이 모델은 빔의 지간-높이 비에 따라서 각각 장단 점이 있으며, 본 논문에 깊은 보를 설계할 때의 팁이 제시되어 있다.
\end{abstract}

Key Words : Deep beam, Strut-and-tie(STM), Clear span-to-depth ratio, ACI

\section{Introduction}

Reinforced Concrete (RC) Deep beam is an important structure element which its design method is one of the most popular subjects in research. But there are recommendation on clear span-to-depth ratio of deep beam except in ACI which states the clear span range for deep beam condition, Particularly in this paper, we conduct a research on deep beam for its appropriate clear span-to-depth ratio compatible with design method of ACI Traditional and STM. In ACI 318-99M [1], clause 11.8-Special provisions for deep flexural members, RC deep beams as of deep flexures are designed by nonlinear distribution of strain and it provides specific shear strength of nominal shear strength of concrete $\left(v_{c}\right)$ and nominal shear strength

${ }^{*}$ Corresponding Author : Byung-Jik Son (Konyang University) Tel: +82-41-730-5634 email: strustar@konyang.ac.kr 
provided by shear reinforcement $\left(v_{s}\right)$ and minimum requirement of shear reinforcement perpendicular to flexural tension reinforcement $\left(A_{v}\right)$ and area of shear reinforcement parallel to flexural tension reinforcement $\left(A_{v h}\right)$ in clause 11.8 .7 through 11.8 .10 respectively whereas later version of ACI 318M [2] do not provide specific provision of $v_{c}$ and $v_{s}$ for deep beams as deep flexures. Only area of shear reinforcement $\left(A_{v}\right)$ and $\left(A_{v h}\right)$ are provided in clause 11.7.4.1 and 11.7.4.2 respectively. However, RC deep beam still can be design by ACI flexure design and in this study we base on ACI 318M-11 [2] accept design of $\left(v_{c}\right)$ and $\left(v_{s}\right)$ that are based on ACI 318M-99 [1]. More over this design is still taken into account for many designers and researchers in field of study and research. Another design method to be mentioned in this study is Strut-and-Tie Models (STM). This model is recognized in ACI from version of ACI 318M-02, STM is partly described in appendix $\mathrm{A}$, and numerous investigations and modifications of the method have been performed. A STM reduces complex states of stress within a D-region of a reinforced or prestressed concrete member into a truss comprised of simple, uniaxial stress paths. Each uniaxial stress path is considered a member of the STM. It considers the complete flow of forces rather than the forces at any one particular section [3]. The internal load path in cracked reinforced concrete is approximated by an idealized truss, where zones of concrete with primarily unidirectional compressive stresses are modeled by compression strut, tension ties are used to model the principal reinforcement, and nodal zones are typically determined by finding the product of the concrete compressive strength and a reduction factor [6]. STM do not separate flexural and shear design, unlike ACI Traditional design. The two methods have its relationships in term of beam's size, and that should be another significant starting point for further investigations.

\section{Deep beam design}

Before starting design a deep beam, first a beam need to checked for deep beam condition basing on ACI the maximum length-to-depth ratio such a D-region would be approximately 2 , clear spans equal to or less than four times overall member depth or regions with concentrated loads within a distance $2 \mathrm{H}$ [2] from the face of support or another word shear span less 2 times of depth. Below are two methods which are mentioned above of ACI Traditional and STM.

\subsection{ACl Traditional design}

First, design flexural reinforcement Eq.1 (ACI R9.1) and compare with minimum of steel requirement provided by (ACI 10.5) Eq.2. Second, design shear reinforcement which are not specific provided by ACI from version of 2002. Strength of concrete $\left(v_{c}\right)$ and strength of steel $\left(v_{s}\right)$ here are quoted from ACI 318-99, section 10.5. In case $\left(v_{s}\right)$ is required, it should done by Eq. 4. Although, by calculation, $\left(v_{s}\right)$ is not required, it should be provided by $A_{s, \min }$.

$$
\begin{aligned}
& M_{u} \leq \phi M_{n}, M_{n}=A_{s} f_{y}\left(d-\frac{a}{2}\right) \\
& =A_{s} f_{y}\left(d-\frac{A_{s} f_{y}}{2 \times 0.85 f_{c k} b_{w}}\right) \\
& A_{s}>A_{s, \min }=\frac{0.25 \sqrt{f^{\prime}}}{f_{y}} b_{w} d>1.4 \frac{b_{w} d}{f_{y}}
\end{aligned}
$$

\subsection{Strut-and-Tie Model}

First, establish the strut-and-tie model and determine the required truss forces as shown in Fig.1. Second, check bearing capacity at every node and strut. Finally, determine reinforcement. For tie element, main rebar are provided, whereas, strength of struts are used 
for shear reinforcement. Check the anchorage should be done and in case available length of anchorage is smaller than require anchorage, hook must be use. But in reality, hook shall be used for main rebar, so although available of anchorage is bigger than require anchorage hook should be used and since we try to have the result of steel requirement is accurate with the real of construction, all main rebar are included with hook [4].

\section{Parameter study}

In this study, there are two parameters of overall depth of beam and load location, see Table 1 . Overall depth of beam starts from $1.1 \mathrm{~m}$ to $2 \mathrm{~m}$ (10 variations) and load location (from center of support) varies from $1.1 \mathrm{~m}$ to $2 \mathrm{~m}$ (10 variations). Each parameter of overall depth of beams are fixed and combine with parameter of load location. These parameters consist of 100 samples but since 32 beams are not considered as deep beam by ACI, so only

[Table 1] Beam dimensions (m) and material properties (MPa)

\begin{tabular}{|c|c|c|c|c|c|c|c|c|}
\hline$l_{n}$ & $s_{x}$ & $c_{x}$ & $b_{w}$ & $H$ & $l_{1}$ & $f^{\prime}$ & $f_{y}$ & $p_{u}{ }^{(\mathrm{kN})}$ \\
\hline 3.6 & 0.5 & 0.5 & 0.5 & $1.1-2$ & $1.1-2$ & 28 & 410 & 2500 \\
\hline
\end{tabular}

68 result of steel requirement in shear (vertical and horizontal steel) and in moment (main rebar) are used to be analyzed.

Excel VBA is used for added design so the results should be acceptable. However, since the results are taken from the final design, used for real construction, we must accept that some variations make less difference. For instant, slight difference in shear reinforcements may have the same amount of steel after reinforcement arrangement.

\section{Results and discussion}

Our purpose is to compare results which taken from the final design. So instead of finding ultimate shear failure, the results of steel requirements (reinforcement arrangement) are taken to be analyzed. In average among 68 samples, STM require total of steel more than ACI Traditional about 1.1 times for total steel, 1.03 times for stirrup and 1.15 times for main rebar. Deep beams change in overall depth of beams when clear span-to-depth ratio $\left(l_{n} / H\right)$ is larger than 2.6, it is not adequate with bearing capacity for STM since bearing capacity is not satisfy by code requirement.

For ACI Traditional it shows an abnormal results of

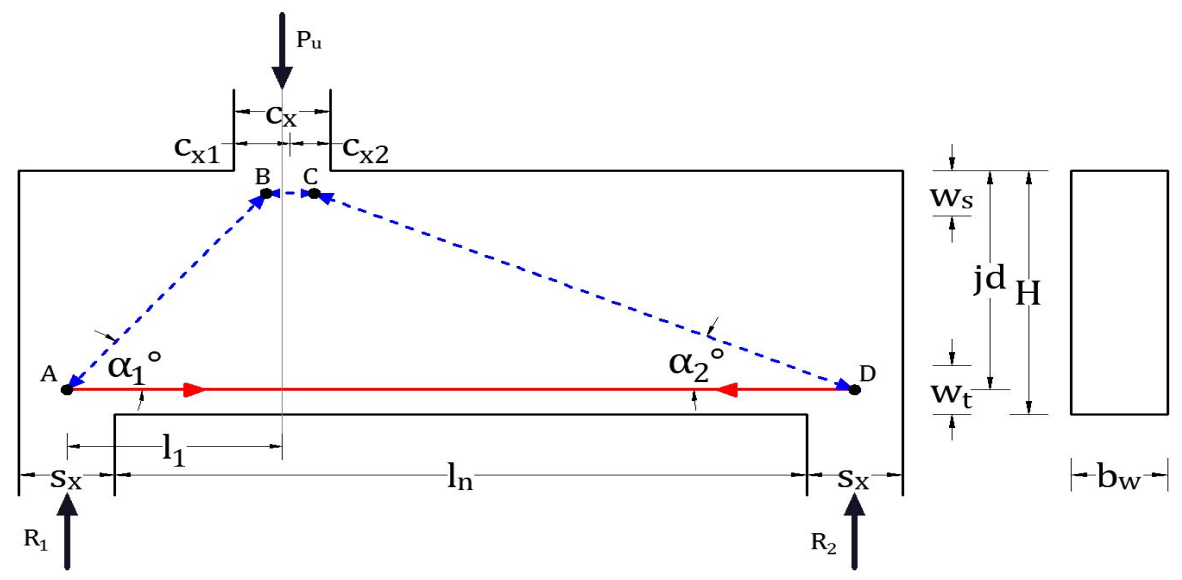

[Fig. 1] Strut-and-Tie Model in deep beam 
dropping down from a high requirement of steel as shown in Fig.5. When clear span-to-depth ratio $\left(l_{n} / H\right)$ is larger than 2.4, ACI Traditional and STM are far different while clear span-to-depth ratio $\left(l_{n} / H\right)$ equal to 2.3 both design methods show appropriate result of increasing of which has its clear span-to-depth ratio of 2.3 or nearest points (can be from 2.4) should be the most appropriate deep beam for both ACI Traditional and STM.

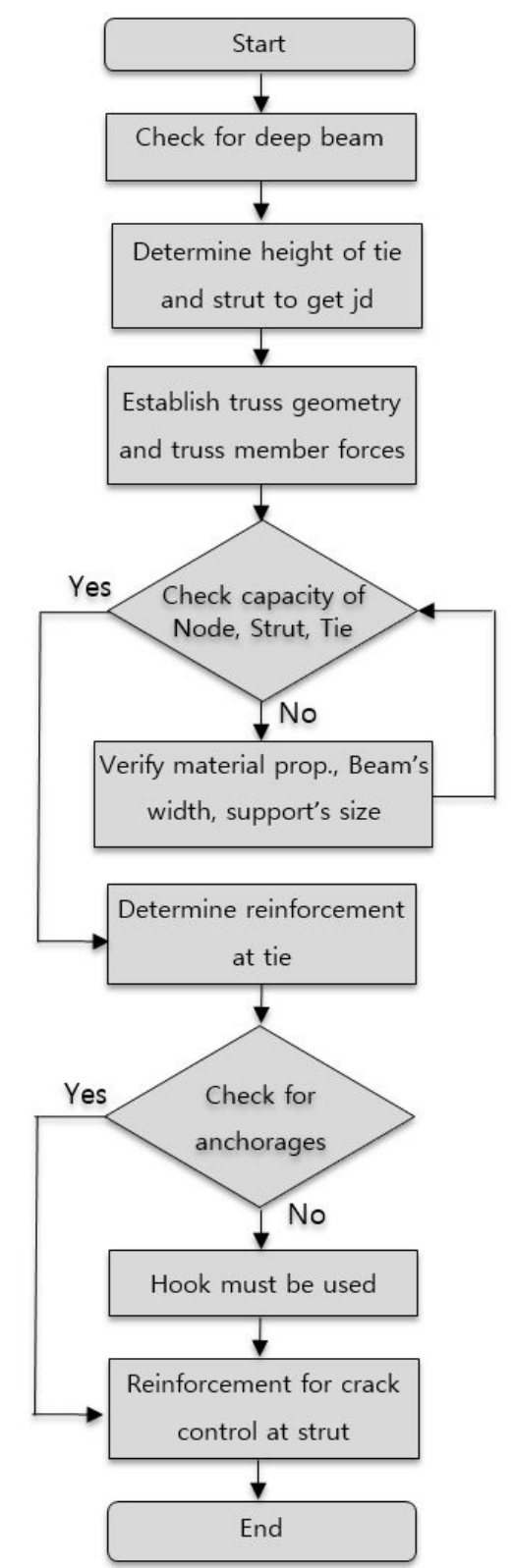

[Fig. 2] Design chart flow;

(a) ACI Traditional design chart flow, (b) STM, design chart flow 
[Table 2] Result of steel provided

\begin{tabular}{|c|c|c|c|c|c|c|c|c|c|c|c|c|c|}
\hline \multirow{2}{*}{$l_{n}$} & \multicolumn{5}{|c|}{ Volume of steel $\mathrm{cm}^{3}$ (ACI Traditional) } & \multicolumn{5}{|c|}{ Volume of steel $\mathrm{cm}^{3}$ (STM) } & \multicolumn{3}{|c|}{ STM/Traditional } \\
\hline & $v_{h}$ & $v_{v}$ & $v_{\text {stirrup }}$ & $v_{\text {main }}$ & $v_{\text {total }}$ & $v_{h}$ & $v_{v}$ & $v_{\text {stirrup }}$ & $v_{\text {main }}$ & $v_{\text {total }}$ & $v_{\text {stirrup }}$ & $v_{\text {main }}$ & $v_{\text {total }}$ \\
\hline \multicolumn{14}{|c|}{$\mathrm{H}=1.4 \mathrm{~m}$} \\
\hline 1.3 & $13,172.8$ & $13,784.2$ & $26,957.0$ & $26,620.1$ & $53,577.1$ & 6,973.4 & $7,952.4$ & $14,925.8$ & $31,460.1$ & $46,385.9$ & 0.55 & 1.18 & 0.87 \\
\hline 1.4 & $13,172.8$ & $13,784.2$ & $26,957.0$ & $29,040.1$ & $55,997.1$ & $6,973.4$ & $7,952.4$ & $14,925.8$ & $33,880.1$ & $48,805.9$ & 0.55 & 1.17 & 0.87 \\
\hline 1.5 & $11,709.1$ & $12,723.9$ & $24,433.1$ & $29,040.1$ & $53,473.1$ & 6,973.4 & $7,952.4$ & $14,925.8$ & $33,880.1$ & $48,805.9$ & 0.61 & 1.17 & 0.91 \\
\hline 1.6 & $11,709.1$ & $12,723.9$ & $24,433.1$ & $29,040.1$ & $53,473.1$ & 6,973.4 & $7,952.4$ & $14,925.8$ & $36,300.1$ & $51,225.9$ & 0.61 & 1.25 & 0.96 \\
\hline 1.7 & $11,709.1$ & $12,723.9$ & 24,433.1 & $31,460.1$ & $55,893$. & 6,973.4 & $7,952.4$ & $14,925.8$ & $36,300.1$ & $51,225.9$ & 0.61 & 1.15 & 0.92 \\
\hline 1.8 & $11,709.1$ & $12,723.9$ & $24,433.1$ & $31,460.1$ & $55,893.2$ & 6,973.4 & $7,952.4$ & $14,925.8$ & $36,300.1$ & $51,225.9$ & 0.61 & 1.15 & 0.92 \\
\hline 1.9 & $11,709.1$ & $12,723.9$ & $24,433.1$ & $31,460.1$ & $55,893.2$ & $6,973.4$ & $7,952.4$ & $14,925.8$ & $38,720.1$ & $53,645.9$ & 0.61 & 1.23 & 0.96 \\
\hline 2.0 & $11,709.1$ & $12,193.7$ & $23,902.9$ & $31,460.1$ & $55,362.9$ & 6,973.4 & $7,952.4$ & $14,925.8$ & $38,720.1$ & $53,645.9$ & 0.62 & 1.23 & 0.97 \\
\hline \multicolumn{14}{|c|}{$\mathrm{H}=1.5 \mathrm{~m}$} \\
\hline 1.1 & $10,245.5$ & $11,219.0$ & $21,464.5$ & $24,200.1$ & $45,664.6$ & 8,368.1 & $8,414.3$ & $16,782.3$ & $26,620.1$ & $43,402.4$ & 0.78 & 1.10 & 0.95 \\
\hline 1.2 & $10,245.5$ & $11,219.0$ & $21,464.5$ & $24,200.1$ & $45,664.6$ & $8,368.1$ & $8,414.3$ & $16,782.3$ & $26,620.1$ & $43,402.4$ & 0.78 & 1.10 & 0.95 \\
\hline 1.3 & $10,245.5$ & $11,219.0$ & $21,464.5$ & $24,200.1$ & $45,664.6$ & 8,368.1 & $8,414.3$ & $16,782.3$ & $29,040.1$ & $45,822.4$ & 0.78 & 1.20 & 1.00 \\
\hline 1.4 & $10,245.5$ & $11,219.0$ & $21,464.5$ & $26,620.1$ & $48,084.6$ & $8,368.1$ & $8,414.3$ & $16,782.3$ & $29,040.1$ & $45,822.4$ & 0.78 & 1.09 & 0.95 \\
\hline 1.5 & $10,245.5$ & $11,219.0$ & $21,464.5$ & $26,620.1$ & $48,084.6$ & 8,368.1 & $8,414.3$ & $16,782.3$ & $31,460.1$ & $48,242.4$ & 0.78 & 1.18 & 1.00 \\
\hline 1.6 & $10,245.5$ & $11,779.9$ & 22,025.4 & $26,620.1$ & $48,645.5$ & $8,368.1$ & $8,414.3$ & $16,782.3$ & $31,460.1$ & $48,242.4$ & 0.76 & 1.18 & 0.99 \\
\hline 1.7 & $10,245.5$ & $10,658.1$ & $20,903.6$ & $29,040.1$ & 49,943.7 & 8,368.1 & $8,414.3$ & $16,782.3$ & $33,880.1$ & $50,662.4$ & 0.80 & 1.17 & 1.01 \\
\hline 1.8 & $10,245.5$ & $10,658.1$ & $20,903.6$ & $29,040.1$ & 49,943.7 & $8,368.1$ & $8,414.3$ & $16,782.3$ & $33,880.1$ & $50,662.4$ & 0.80 & 1.17 & 1.01 \\
\hline 1.9 & $10,245.5$ & $10,097.1$ & $20,342.6$ & $29,040.1$ & $49,382.7$ & $8,368.1$ & $8,414.3$ & $16,782.3$ & $33,880.1$ & $50,662.4$ & 0.82 & 1.17 & 1.03 \\
\hline 2.0 & $10,245.5$ & $10,097.1$ & $20,342.6$ & $29,040.1$ & $49,382.7$ & $8,368.1$ & $8,414.3$ & $16,782.3$ & $33,880.1$ & $50,662.4$ & 0.82 & 1.17 & 1.03 \\
\hline \multicolumn{14}{|c|}{$\mathrm{H}=1.6 \mathrm{~m}$} \\
\hline 1.1 & $8,781.9$ & 8,876.1 & $17,657.9$ & $21,780.1$ & $39,438.0$ & $8,368.1$ & $8,876.1$ & $17,244.1$ & $24,200.1$ & $41,444.2$ & 0.98 & 1.11 & 1.05 \\
\hline 1.2 & $8,781.9$ & 8,876.1 & $17,657.9$ & $21,780.1$ & $39,438.0$ & $8,368.1$ & $8,876.1$ & $17,244.1$ & $24,200.1$ & $41,444.2$ & 0.98 & 1.11 & 1.05 \\
\hline 1.3 & $8,781.9$ & 8,876.1 & $17,657.9$ & $21,780.1$ & $39,438.0$ & $8,368.1$ & $8,876.1$ & $17,244.1$ & $26,620.1$ & 43,864.2 & 0.98 & 1.22 & 1.11 \\
\hline 1.4 & $8,781.9$ & $9,467.8$ & $18,249.7$ & $24,200.1$ & $42,449.7$ & $8,368.1$ & $8,876.1$ & $17,244.1$ & $26,620.1$ & $43,864.2$ & 0.94 & 1.10 & 1.03 \\
\hline 1.5 & $8,781.9$ & 8,876.1 & $17,657.9$ & $24,200.1$ & $41,858.0$ & 8,368.1 & $8,876.1$ & $17,244.1$ & $29,040.1$ & $46,284.2$ & 0.98 & 1.20 & 1.11 \\
\hline 1.6 & $8,781.9$ & 8,876.1 & $17,657.9$ & $24,200.1$ & $41,858.0$ & $8,368.1$ & $8,876.1$ & $17,244.1$ & $29,040.1$ & $46,284.2$ & 0.98 & 1.20 & 1.11 \\
\hline 1.7 & $8,781.9$ & 8,876.1 & $17,657.9$ & $26,620.1$ & $44,278.0$ & 8,368.1 & $8,876.1$ & $17,244.1$ & $29,040.1$ & $46,284.2$ & 0.98 & 1.09 & 1.05 \\
\hline 1.8 & $8,781.9$ & 8,284.3 & $17,066.2$ & $26,620.1$ & $43,686.3$ & $8,368.1$ & $8,876.1$ & $17,244.1$ & $31,460.1$ & $48,704.2$ & 1.01 & 1.18 & 1.11 \\
\hline 1.9 & $8,781.9$ & 8,876.1 & $17,657.9$ & $26,620.1$ & $44,278.0$ & $8,368.1$ & $8,876.1$ & $17,244.1$ & $31,460.1$ & $48,704.2$ & 0.98 & 1.18 & 1.10 \\
\hline 2.0 & $8,781.9$ & 8,284.3 & $17,066.2$ & $26,620.1$ & $43,686.3$ & $8,368.1$ & $8,876.1$ & $17,244.1$ & $31,460.1$ & $48,704.2$ & 1.01 & 1.18 & 1.11 \\
\hline \multicolumn{14}{|c|}{$\mathrm{H}=1.7 \mathrm{~m}$} \\
\hline 1.1 & 7,318.2 & 7,470.3 & $14,788.5$ & $21,780.1$ & $36,568.6$ & $8,368.1$ & $9,337.9$ & $17,705.9$ & $21,780.1$ & $40,880.7$ & 1.20 & 1.00 & 1.12 \\
\hline 1.2 & 7,318.2 & 7,470.3 & $14,788.5$ & 21,780.1 & $36,568.6$ & 8,368.1 & $9,337.9$ & $17,705.9$ & $24,200.1$ & $41,906.0$ & 1.20 & 1.11 & 1.15 \\
\hline 1.3 & 7,318.2 & 8,092.8 & $15,411.1$ & $21,780.1$ & 37,191.1 & $8,368.1$ & $9,337.9$ & $17,705.9$ & $24,200.1$ & $41,906.0$ & 1.15 & 1.11 & 1.13 \\
\hline 1.4 & 7,318.2 & 7,470.3 & $14,788.5$ & $21,780.1$ & $36,568.6$ & 8,368.1 & 9,337.9 & $17,705.9$ & $24,200.1$ & $41,906.0$ & 1.20 & 1.11 & 1.15 \\
\hline 1.5 & $7,318.21$ & 7,470.3 & $14,788.5$ & $21,780.1$ & $36,568.6$ & 8,368.1 & $9,337.9$ & $17,705.9$ & $26,620.1$ & $44,326.0$ & 1.20 & 1.22 & 1.21 \\
\hline 1.6 & 7,318.2 & 8,092.8 & 15,411.1 & $24,200.1$ & 39,611.1 & $8,368.1$ & $9,337.9$ & $17,705.9$ & 26,620.1 & $44,326.0$ & 1.15 & 1.10 & 1.12 \\
\hline 1.7 & 7,318.2 & 7,470.3 & $14,788.5$ & $24,200.1$ & 38,988.6 & 8,368.1 & $9,337.9$ & $17,705.9$ & $26,620.1$ & $44,326.0$ & 1.20 & 1.10 & 1.14 \\
\hline 1.8 & 7,318.2 & 7,470.3 & $14,788.5$ & $24,200.1$ & 38,988.6 & 8,368.1 & 9,337.9 & $17,705.9$ & $29,040.1$ & $46,746.1$ & 1.20 & 1.20 & 1.20 \\
\hline 1.9 & 7,318.2 & 8,092.8 & 15,411.1 & $24,200.1$ & 39,611.1 & $8,368.1$ & 9,337.9 & $17,705.9$ & $29,040.1$ & $46,746.1$ & 1.15 & 1.20 & 1.18 \\
\hline 2.0 & 7,318.2 & $7,470.3$ & $14,788.5$ & $24,200.1$ & $38,988.6$ & 8,368.1 & $9,337.9$ & $17,705.9$ & $29,040.1$ & $46,746.1$ & 1.20 & 1.20 & 1.20 \\
\hline
\end{tabular}


Design Comparison of Strut Tie Model and ACI Traditional by Clear Span-to-Depth Ratio

\begin{tabular}{|c|c|c|c|c|c|c|c|c|c|c|c|c|c|}
\hline \multicolumn{14}{|c|}{$\mathrm{H}=1.8 \mathrm{~m}$} \\
\hline 1.1 & $8,781.9$ & $7,839.8$ & $16,621.6$ & $19,360.1$ & $35,981.7$ & $9,762.8$ & $9,799.7$ & $19,562.5$ & $19,360.1$ & $38,922.5$ & 1.18 & 1.00 & 1.08 \\
\hline 1.2 & $8,781.9$ & $7,839.8$ & $16,621.6$ & $19,360.1$ & $35,981.7$ & $9,762.8$ & $9,799.7$ & $19,562.5$ & $21,780.1$ & $41,342.5$ & 1.18 & 1.13 & 1.15 \\
\hline 1.3 & $8,781.9$ & $8,493.1$ & $17,274.9$ & $19,360.1$ & $36,634.9$ & $9,762.8$ & $9,799.7$ & $19,562.5$ & $21,780.1$ & $41,342.5$ & 1.13 & 1.13 & 1.13 \\
\hline 1.4 & $8,781.9$ & $7,839.8$ & $16,621.6$ & $21,780.1$ & $38,401.7$ & $9,762.8$ & $9,799.7$ & $19,562.5$ & $24,200.1$ & $43,762.5$ & 1.18 & 1.11 & 1.14 \\
\hline 1.5 & $8,781.9$ & $7,839.8$ & $16,621.6$ & $21,780.1$ & $38,401.7$ & $9,762.8$ & $9,799.7$ & $19,562.5$ & $24,200.1$ & $43,762.5$ & 1.18 & 1.11 & 1.14 \\
\hline 1.6 & $8,781.9$ & 8,493.1 & $17,274.9$ & $21,780.1$ & $39,055.0$ & $9,762.8$ & $9,799.7$ & $19,562.5$ & $24,200.1$ & $43,762.5$ & 1.13 & 1.11 & 1.12 \\
\hline 1.7 & $8,781.9$ & $7,839.8$ & $16,621.6$ & $21,780.1$ & $38,401.7$ & $9,762.8$ & $9,799.7$ & $19,562.5$ & $26,620.1$ & $46,182.5$ & 1.18 & 1.22 & 1.20 \\
\hline 1.8 & $8,781.9$ & $7,839.8$ & $16,621.6$ & $21,780.1$ & $38,401.7$ & $9,762.8$ & $9,799.7$ & $19,562.5$ & $26,620.1$ & $46,182.5$ & 1.18 & 1.22 & 1.20 \\
\hline 1.9 & $8,781.9$ & 8,493.1 & $17,274.9$ & $21,780.1$ & $39,055.0$ & $9,762.8$ & $9,799.7$ & $19,562.5$ & $26,620.1$ & $46,182.5$ & 1.13 & 1.22 & 1.18 \\
\hline 2.0 & $8,781.9$ & $7,839.8$ & $16,621.6$ & $21,780.1$ & $38,401.7$ & $9,762.8$ & $9,799.7$ & $19,562.5$ & $26,620.1$ & $46,182.5$ & 1.18 & 1.22 & 1.20 \\
\hline \multicolumn{14}{|c|}{$\mathrm{H}=1.9 \mathrm{~m}$} \\
\hline 1.1 & $8,781.9$ & 8,209.2 & 16,991.1 & $19,360.1$ & $36,351.1$ & $9,762.8$ & $10,261.5$ & $20,024.3$ & $19,360.1$ & $39,384.3$ & 1.18 & 1.00 & 1.08 \\
\hline 1.2 & $8,781.9$ & 8,209.2 & 16,991.1 & $19,360.1$ & $36,351.1$ & $9,762.8$ & $10,261.5$ & $20,024.3$ & $19,360.1$ & $39,384.3$ & 1.18 & 1.00 & 1.08 \\
\hline 1.3 & $8,781.9$ & 8,893.3 & $17,675.2$ & $19,360.1$ & $37,035.2$ & $9,762.8$ & $10,261.5$ & $20,024.3$ & $21,780.1$ & $41,804.3$ & 1.13 & 1.13 & 1.13 \\
\hline 1.4 & $8,781.9$ & $8,209.2$ & 16,991.1 & $19,360.1$ & $36,351.1$ & $9,762.8$ & $10,261.5$ & $20,024.3$ & $21,780.1$ & $41,804.3$ & 1.18 & 1.13 & 1.15 \\
\hline 1.5 & $8,781.9$ & $8,209.2$ & $16,991.1$ & $19,360.1$ & $36,351.1$ & $9,762.8$ & $10,261.5$ & $20,024.3$ & $24,200.1$ & $44,224.3$ & 1.18 & 1.25 & 1.22 \\
\hline 1.6 & $8,781.9$ & 8,893.3 & $17,675.2$ & $21,780.1$ & $39,455.2$ & $9,762.8$ & $10,261.5$ & $20,024.3$ & $24,200.1$ & $44,224.3$ & 1.13 & 1.11 & 1.12 \\
\hline 1.7 & $8,781.9$ & 8,209.2 & 16,991.1 & $21,780.1$ & $38,771.1$ & $9,762.8$ & $10,261.5$ & $20,024.3$ & $24,200.1$ & $44,224.3$ & 1.18 & 1.11 & 1.14 \\
\hline 1.8 & $8,781.9$ & $8,209.2$ & $16,991.1$ & $21,780.1$ & $38,771.1$ & $9,762.8$ & $10,261.5$ & $20,024.3$ & $24,200.1$ & $44,224.3$ & 1.18 & 1.11 & 1.14 \\
\hline 1.9 & $8,781.9$ & 8,893.3 & $17,675.2$ & $21,780.1$ & $39,455.2$ & $9,762.8$ & $10,261.5$ & $20,024.3$ & $24,200.1$ & $44,224.3$ & 1.13 & 1.11 & 1.12 \\
\hline 2.0 & $8,781.9$ & $8,209.2$ & 16,991.1 & $21,780.1$ & $38,771.1$ & $9,762.8$ & $10,261.5$ & $20,024.3$ & $24,200.1$ & $44,224.3$ & 1.18 & 1.11 & 1.14 \\
\hline \multicolumn{14}{|c|}{$\mathrm{H}=2.0 \mathrm{~m}$} \\
\hline 1.1 & $8,781.9$ & $8,578.7$ & $17,360.5$ & $16,940.1$ & $34,300.6$ & $11,157.4$ & $10,723.3$ & $21,880.8$ & $19,360.1$ & $41,240.8$ & 1.26 & 1.14 & 1.20 \\
\hline 1.2 & $8,781.9$ & $8,578.7$ & $17,360.5$ & $16,940.1$ & $34,300.6$ & $11,157.4$ & $10,723.3$ & $21,880.8$ & $19,360.1$ & $41,240.8$ & 1.26 & 1.14 & 1.20 \\
\hline 1.3 & $8,781.9$ & $9,293.6$ & $18,075.4$ & $16,940.1$ & $35,015.5$ & $11,157.4$ & $10,723.3$ & $21,880.8$ & 19,360.1 & $41,240.8$ & 1.21 & 1.14 & 1.18 \\
\hline 1.4 & $8,781.9$ & $8,578.7$ & $17,360.5$ & $19,360.1$ & $36,720.6$ & $11,157.4$ & $10,723.3$ & $21,880.8$ & $21,780.1$ & $43,660.8$ & 1.26 & 1.13 & 1.19 \\
\hline 1.5 & $8,781.9$ & $8,578.7$ & $17,360.5$ & $19,360.1$ & $36,720.6$ & $11,157.4$ & $10,723.3$ & $21,880.8$ & $21,780.1$ & $43,660.8$ & 1.26 & 1.13 & 1.19 \\
\hline 1.6 & $8,781.9$ & $9,293.6$ & $18,075.4$ & $19,360.1$ & $37,435.5$ & $11,157.4$ & $10,723.3$ & $21,880.8$ & $21,780.1$ & $43,660.8$ & 1.21 & 1.13 & 1.17 \\
\hline 1.7 & $8,781.9$ & $8,578.7$ & $17,360.5$ & $19,360.1$ & $36,720.6$ & $11,157.4$ & $10,723.3$ & $21,880.8$ & $21,780.1$ & $43,660.8$ & 1.26 & 1.13 & 1.19 \\
\hline 1.8 & $8,781.9$ & $8,578.7$ & $17,360.5$ & $19,360.1$ & $36,720.6$ & $11,157.4$ & $10,723.3$ & $21,880.8$ & $24,200.1$ & $46,080.8$ & 1.26 & 1.25 & 1.25 \\
\hline 1.9 & $8,781.9$ & $9,293.6$ & $18,075.4$ & $19,360.1$ & $37,435.5$ & $11,157.4$ & $10,723.3$ & $21,880.8$ & $24,200.1$ & $46,080.8$ & 1.21 & 1.25 & 1.23 \\
\hline 2.0 & $8,781.9$ & $8,578.7$ & $17,360.5$ & $19,360.1$ & $36,720.6$ & $11,157.4$ & $10,723.3$ & $21,880.8$ & $24,200.1$ & $46,080.8$ & 1.26 & 1.25 & 1.25 \\
\hline
\end{tabular}

\section{Conclusions}

This study can be concluded that deep beam which has its clear span-to-depth ratio $\left(l_{n} / H\right)$ equal to 2.3 should be the most appropriate deep beams which are designed by ACI Traditional and STM. And with this point, it is easier to use ACI Traditional rather than STM by just adding about $10 \%$ of total steel because STM design procedures are more complicated than ACI Traditional and need to spend more time. However, 
STM should be more recommended rather than ACI Traditional for deep beam which has $\left(l_{n} / H\right)$ less than 2.3. ACI Traditional method still can be used but it should be avoided or another words more study on deep beams which have $\left(l_{n} / H\right)$ is greater than 2.3 should be investigated. We hope this small scope of study could be a useful reference for further research.
Finally we strongly recommend that more study on deep for $\left(l_{n} / H\right)>2.3$ should be widely done since this size of ratio can guide us for comparing between ACI Traditional and Strut-tie-model.

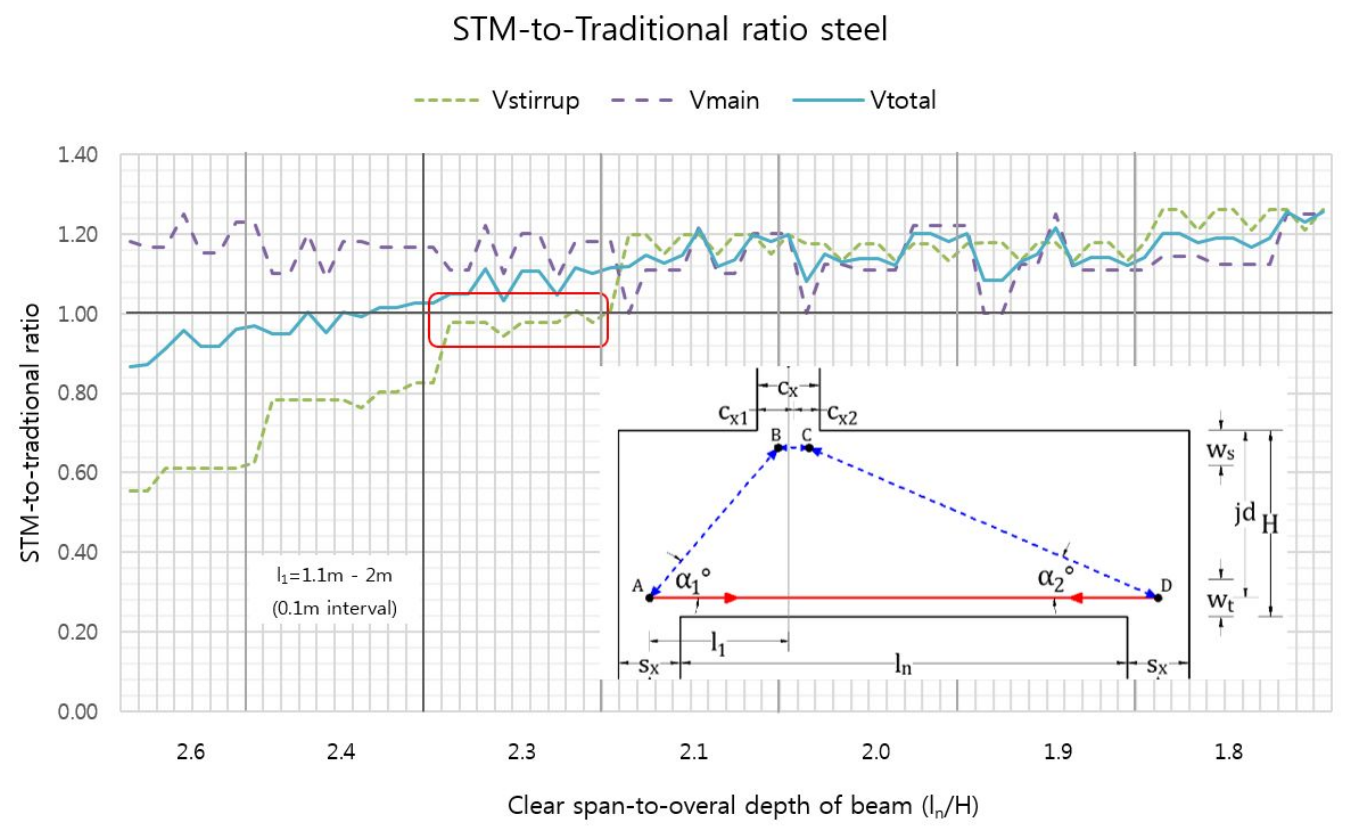

[Fig. 3] STM-to-Traditional ratio steel

Volume of vertical stirrup

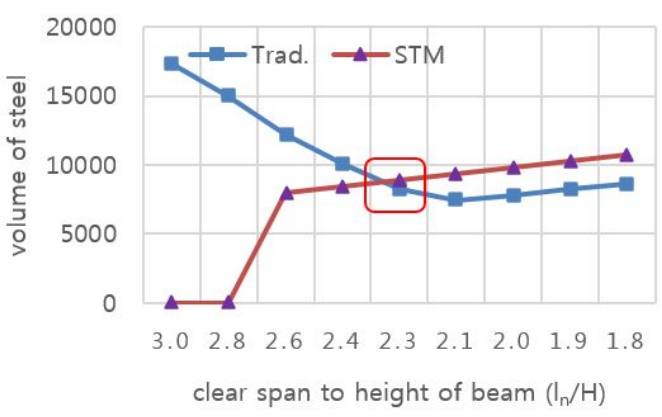

[Fig. 4] Volume of vertical $\operatorname{stirrup}\left(l_{n}=0.2 m\right)$

\section{Volume of total stirrup}

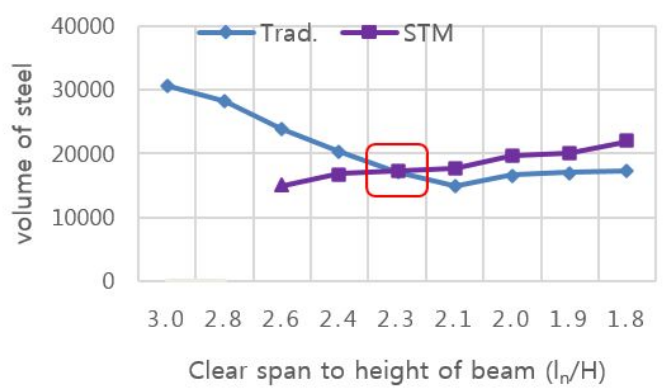

[Fig. 5] Volume of total $\operatorname{stirrup}\left(l_{n}=0.2 m\right)$ 


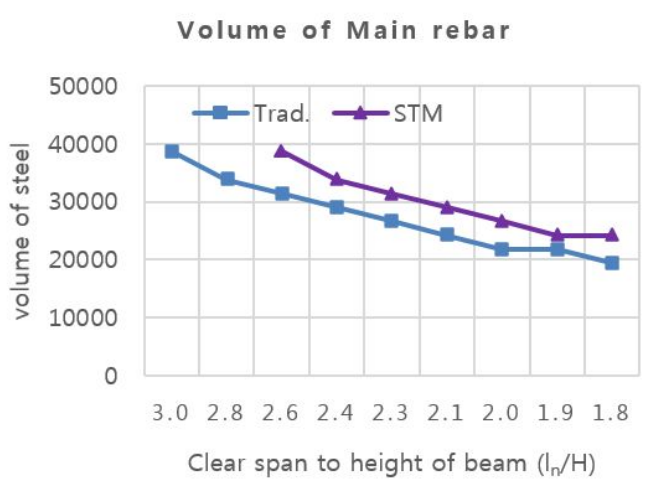

[Fig. 6] Volume of main $\operatorname{rebar}\left(l_{n}=0.2 m\right)$

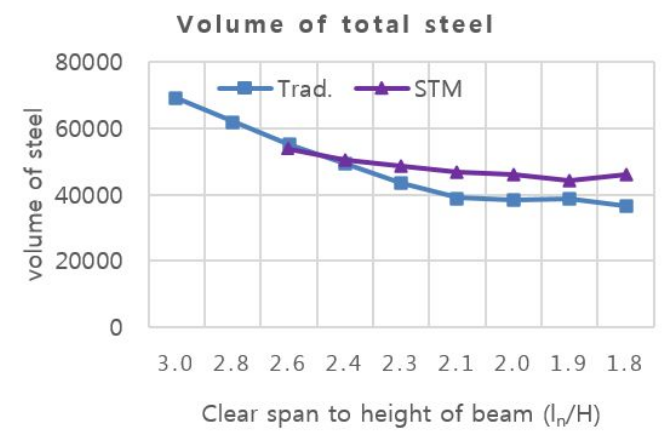

[Fig. 7] Volume of total steel $\left(l_{n}=0.2 m\right)$

\section{References}

[1] ACI Committee 318, 1999, "Building Code Requirements for Structural Concrete (ACI 318M-99) and Commentary (318RM-99)", American Concrete Institute, Farmington Hills, pp.163-164, 1999.

[2] ACI Committee 318, 2011, "Building Code Requirements for Structural Concrete (ACI 318M-11) and Commentary (318RM-11)", American Concrete Institute, Farmington Hills, pp. 387-401, 2011.

[3] James K. Wight and James G. MacGregor, Reinforced Concrete Mechanics and Design, Pearson, Six Edition, pp. 879-971, 2012.

[4] James K. Wight and Gustavo J. Parra-Montesinos, Strut-and-Tie Model for Deep Beam Design, Concrete International.

[5] Karl-Heinz Reineck and Lawrence C. Novak, Further Examples for the Design of Structural Concrete with
Strut-and-Tie Models, 2011.

[6] Verification and implementation of strut-and-tie Model in LRFD Bridge design Specifications, barney $\mathrm{T}$. Martin, Jr., Phd., P.E., November, 2007.

[7] ACI Structural journal, Design of deep beams suing strut-and-tie models part I\&II, Michael D. Brown and Oguzhan Bayrak.

\section{UY LYMEI}

[Regular member]

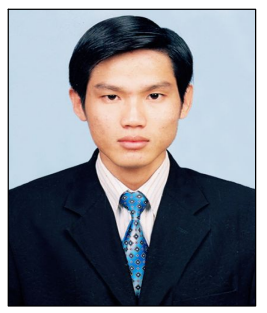

- Nov. 2009 : Norton Univ., Civil Engineering, BEng

- Mar. 2012 current : Konyang Univ., Dept. of Civil and Environmental Engineering, MEng

$<$ Research Interests $>$

Structure analysis, RC, Construction management

\section{Byung-Jik Son}

[Regular member]

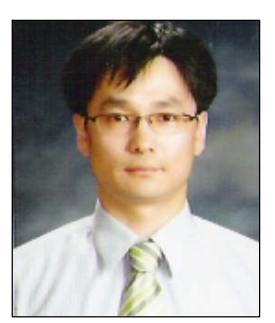

- Feb. 1998 : University of Seoul, Civil Engineering, BEng

- Feb. 2000 : University of Seoul, Civil Engineering, MEng

- Aug. 2003 : University of Seoul, Civil Engineering, $\mathrm{PhD}$

- Mar. 2005 current : Konyang Univ., Dept. of Civil and Environmental Engineering, Porfessor

$<$ Research Interests $>$

FRP (Fiber Reinforcement Plastics) structure analysis, Bridge Engineering, Damage Detection, Earthquake Engineering, DSP (Digital Signal Processing) 